\title{
Operating on Tensors Within Dynamic Coordinate Frames
}

\author{
Keith C. Afas* \\ University of Western Ontario, Department of Medical Sciences \\ London, Ontario, N6A 5B7, CANADA ${ }^{\dagger}$
}

October 18, 2018

\section{Contents}

1 Introduction 3

1.1 The Effect on Existing Physical Laws . . . . . . . . . . . . . 3

1.2 Geometry of the Dynamic Coordinate Frame: . . . . . . . . . 4

1.2.1 Beta-Symbol . . . . . . . . . . . . 5

1.2.2 The Spatiotemporal Commutator . . . . . . . . 6

1.2.3 Beta-Christoffel Relation . . . . . . . . . . . . 7

1.2.4 Spatiotemporal Change of Coordinates . . . . . . . 8

1.3 Transformation of a Partial Time Derivative: Issues Arise . . . 9

1.4 A Similar Issue: The Calculus of Moving Surfaces . . . . . . . 10

1.4.1 Geometry of CMS . . . . . . . . . . . . 10

1.4.2 Transformation of an Surface Invariant . . . . . . . . 10

2 The Temporal Field Derivative 11

2.1 From CMS to Moving Coordinate Frames . . . . . . . . . . . 11

2.2 Invariance Condition . . . . . . . . . . . . . . . . 12

2.2.1 The Lambda Object: Symmetries Arise . . . . . . . . . 12

2.2.2 A General Class of Operators . . . . . . . . . . . 12

2.3 The Directed Temporal Field Derivative . . . . . . . . . . . . 13

2.4 Extending the Derivative to Higher Rank Tensors . . . . . . . 13

*kafas@uwo.ca, ORCID: 0000-0003-4540-7085

${ }^{\dagger}$ Keywords: Dynamic, Tensor, Operator, Moving Coordinates, 4D Space, Invariant 
2.4.1 Introducing the Product Rule for Metrilinicity . . . . . 13

2.4.2 The General Mixed Temporal Field Derivative . . . . . 14

2.4.3 The Metrilinic Property . . . . . . . . . . . . . . 14

2.5 The Temporal Field Derivative's Tensorial Property . . . . . . 15

2.6 Explicit Derivation for a Tensor of Rank $(1,0)$. . . . . . . . . 15

3 First Order Commutations $\quad 16$

3.1 Temporal Field Derivative Commutations . . . . . . . . . . . 16

3.2 Tensorial Identity of the Commutation . . . . . . . . . . . 17

3.2.1 Verification of an earlier Tensorial Statement . . . . . . 18

3.3 Commutation of the Tensorial Operator . . . . . . . . . . . 19

3.3.1 Further Implications of the Condition . . . . . . . . 20

4 Determining the Lambda Object 20

4.1 The Variance Speed . . . . . . . . . . . . . . . . . . . . . 20

4.1.1 The Variance Speed-Beta Symbol's Relationship . . . . 21

4.2 Guessing a Form: The Variance Speed Form . . . . . . . . . . 22

4.3 The Variance Speed \& First Order Commutations . . . . . . . 24

5 Introducing Notions of Spatio-Temporality 25

5.1 Geometry of the 4D Space . . . . . . . . . . . . . 25

5.2 Vanishing of the Invariant Temporal Commutation . . . . . . 26

6 Higher Order Continuum Temporal Differential Calculus 29

6.1 Higher Order Temporal Derivatives . . . . . . . . . . . . . . 29

6.2 Truly Invariant D'Alembertian . . . . . . . . . . . . . . . 30

7 Applications \& Extensions of the Temporal Field Derivative 31

7.1 Wave Dynamics . . . . . . . . . . . . . . . . 31

7.2 Continuum Mechanics . . . . . . . . . . . . . 31

7.3 Quantum Mechanics ... . . . . . . . . . . . 32

7.3.1 The Klein Gordon Equation . . . . . . . . . . . . 33

8 Conclusion $\quad 33$

\section{Abstract}

This paper puts forward an alteration for Tensor Calculus utliized in a coordinate system which is under a dynamic distortion drawing inspiration from similar fields such as the Calculus of Moving Surfaces (CMS). The paper establishes transformation laws for Tensors within these regions and establishes 
Operators such as the Tensorial Field Derivative which enforce a Tensorial Transformation on Tensors defined within a Dynamically Moving coordinate system. This variation of Tensor Calculus can be utilized to observe how disciplines such as QFT and Continuum Mechanics would change to accomodate for a distorting coordinate system and can be utliized to develop new theoretical models which account for this temporal distortion particularly within Biological Settings.

\section{Introduction}

In Physics, several disciplines have gained prominence for proposing powerful theories to describe several of the Sciences [1,2]. Continuum Mechanics, General Relativity, Quantum Mechanics, and Electromagnetism are among popular examples. All these three fields, though describing radically different settings all share one aspect in common; they share that they are formulated in Tensor Calculus $[6,7,12]$.

Tensor Calculus, while powerful has limitations; since these theories rely heavily on Tensor Calculus, and Tensor Calculus relies heavily on assumptions made about our reality [1], several theories proposed by Tensor Calculus can become innacurate under several unique but common circumstances [2].

In 2016, Gravitational Waves were observed for the first time from a Binary Black Hole Merger [8]. This discovery highlighted an interesting aspect to our physical laws: due to the time-dependent local distortion of space that occurs as a result of General Relativity [9], laws defined by Continuum Mechanics or other Physical Theories require a re-examination. Tensor Calculus used within these theories rely on the concept that the basis of the coordinate system being used do not change in time $\partial_{t} \mathbf{Z}_{i}=\mathbf{0}$. Clearly in areas where Gravitational Waves have a strong influence, or any other distorted regions, this will not be true and all the laws which describe nature under these dynamic conditions need to be reformulated.

\subsection{The Effect on Existing Physical Laws}

Often many physical laws formulated tensorially contain standard time derivatives such as in Continuum Mechanics and Quantum Field Theory (QFT). For example, in Continuum Mechanics, Cauchy's First Law of Motion from an Eulerian Coordinate Frame takes the following Index Notation [2, 6]:

$$
\rho \partial_{t} v^{i}=\rho b^{i}+\nabla_{j} \sigma^{j i}
$$


Where $\rho$ is the density of the continuum, $v^{i}$ is referred to as the tensorial velocity of the continua, $b^{i}$ is the tensorial component of a body force, and $\sigma^{i j}$ is the Cauchy Stress Tensor in component form. Here, $\partial_{t}$ refers to the partial derivative with respect to time (ie. $\partial_{t}=\frac{\partial}{\partial t}$ ); this convention will be used throughout. In QFT, the celebrated Schrodinger Equation used to determine laws of action on a wavefunction is typically given in the following form[12]:

$$
i \hbar \partial_{t} \Psi=\left(-\frac{\hbar^{2}}{2 \mu} \nabla_{i} \nabla^{i}+V\right) \Psi
$$

Both these equations, though they may obey Galilean/Special Relativity $[6,7,12]$, serious issues arise with the preservation of Tensorial properties even for scalars (tensors of rank zero) when the underlying coordinate system is under a temporal deformation induced by General Relativistic Effects. This can be observed by considering the standard time derivative of a Tensor Field of rank $(0,0)$.

\subsection{Geometry of the Dynamic Coordinate Frame:}

As a forenote, often in disciplines such as CMS, indices are often omitted when demostrated in the arguments of functions: this convention will be used throughout the article; Einstein Summation Convention will also be used throughout the length of the document.

Consider a standard coordinate system (3D \& Time), this can be modelled as such: $\left(t, Z^{1}, Z^{2}, Z^{3}\right) \rightarrow(t, Z)$, where $Z=\left(Z^{1}, Z^{2}, Z^{3}\right)$ are the coordinates of the ambient space. In general, given components $\xi^{i}$ pertaining to a arbitrary position, $\mathbf{R}$, the position of a particle can be expressed as [1]:

$$
\mathbf{R}=\xi^{i} \mathbf{Z}_{i}
$$

Where $\mathbf{Z}_{i}$ are the basis vectors for the coordinate system. In general, basis vectors are assumed to depend on the ambient coordinate space $\mathbf{Z}_{i}(Z)$, and this dependency is well known to induce Christoffel Symbols in the Space given as such:

$$
\partial_{j} \mathbf{Z}_{i}=\Gamma_{i j}^{k} \mathbf{Z}_{k}
$$

Where, $\partial_{i}$ refers to the partial derivative with respect to Ambient Coordinates $Z=\left(Z^{1}, Z^{2}, Z^{3}\right)$ in the sense that $\partial_{i}=\frac{\partial}{\partial Z^{i}}$. This denotion will also be used alongside the "comma" notation for partial differentiation. This is summarized by the following:

$$
\frac{\partial}{\partial Z^{i}} T=\partial_{i} T=T_{, i}
$$


The comma shorthand refers to partial differentiation according to the Ricci convention $[1,4,6]$. These two notations will be used throughout

\subsubsection{Beta-Symbol}

In a Dynamic Coordinate System, curvilinear variance will not be the only variance existing in the space [5]. For a dynamic coordinate system system, in full generality, the basis vectors will take the following form:

$$
\mathbf{Z}_{i}=\mathbf{Z}_{i}(t, Z)
$$

Thus, in addition to the curvilinear variance outlined in Eq.(3), the temporal variance in the coordinate frame must also be considered $[1,5]$. This variance will be denoted as the Dynamic Beta-Symbol:

$$
\partial_{t} \mathbf{Z}_{i}=\beta_{i}^{j} \mathbf{Z}_{j}
$$

Much like the Christoffel Symbols, this can be realized in terms of the basis vectors, as well as in terms of the Metric Tensor of the space [1]. In terms of the basis vectors the symbol is given by:

$$
\beta_{j}^{i}=\mathbf{Z}^{i} \cdot \partial_{t} \mathbf{Z}_{j}
$$

Here it is assumed that the indices of the Beta-Symbols can be lowered by the metric tensor. To ensure that the positioning of the indices is not compromised, the dot notation will be introduced to denote specific lowered and raised indices $\left(\beta_{i j}{ }_{i j}=Z_{i k} \beta_{j}^{k}\right)$. Thus, recalling the definition of the metric tensor $Z_{i j}=\mathbf{Z}_{i} \cdot \mathbf{Z}_{j}$, and using Eq.(5), the derivative of the metric tensor yields:

$$
\partial_{t} Z_{i j}=2 \beta_{(i j)}^{\bullet}
$$

Where the symmetric, and antisymmetric component of a tensor are defined by the following two operations $[2,4,6]$ :

$$
A_{(i j)}=\frac{1}{2}\left(A_{i j}+A_{j i}\right), A_{[i j]}=\frac{1}{2}\left(A_{i j}-A_{j i}\right)
$$

This formula can also be extended to the Inverse Metric Tensor:

$$
\partial_{t} Z^{i j}=-2 \beta^{(i j)}
$$

At this point, based on the Inverse Metric Tensor's definition, a similar type of relation can be defined in two different ways for the contravariant basis 
which appear to both satisfy the Inverse Metric Tensor's formula, but do not appear equivalent because of the strict form of the Beta-Symbol:

$$
\partial_{t} \mathbf{Z}^{i}=-\beta^{i}{ }_{j} \mathbf{Z}^{j}, \partial_{t} \mathbf{Z}^{i}=-\beta_{j}^{\bullet} \mathbf{Z}^{j}
$$

Which one is correct? This can be determined by observing the explicit definition of the Beta-Symbols. Due to the symmetrization of the Metric Tensor [4], and the vanishing of the time derivative of the Kronecker Delta [1], the Beta Symbol has the explicit form of:

$$
\beta_{j}^{i}=\frac{1}{2} Z^{i k} \partial_{t} Z_{k j}
$$

Based on this, using the definition $\left(\beta_{j}^{\bullet} i \cdot=Z_{j k} Z^{i m} \beta_{m}^{k}\right)$, and the explicit definition of the Beta-Symbols, the following relation is obtained:

$$
\beta_{j}^{\bullet}{ }^{i}=\frac{1}{2} Z_{j k} Z^{i m} Z^{k p} \partial_{t} Z_{p m}=\frac{1}{2} Z^{i m} \partial_{t} Z_{j m}=\beta^{i}{ }_{j}
$$

Therefore, the Beta-symbol is symmetric in that the order of its indices do not matter! This implies the relieving result that both of the definitions of the Contravariant Basis' time derivative are correct. Therefore, the Beta Symbol can be re-written without reference to index position $\left(\beta_{j}^{i}\right)$; this implies that the Beta Symbol has three symmetric forms obtained through the raising and lowering of indices: $\beta_{j}^{i}$, $\beta^{i j}$, and $\beta_{i j}$. Thus, for the bases of the Ambient Space:

$$
\partial_{t} \mathbf{Z}_{i}=\beta_{i}^{j} \mathbf{Z}_{j}, \partial_{t} \mathbf{Z}^{i}=-\beta_{j}^{i} \mathbf{Z}^{j}
$$

And thankfully so, the Derivatives of the Metric Tensors reduce to:

$$
\partial_{t} Z_{i j}=2 \beta_{i j}, \partial_{t} Z^{i j}=-2 \beta^{i j}
$$

Interestingly, the Beta-symbol is seen to vanish if the coordinate frames are not moving:

$$
\beta_{j}^{i} \approx 0
$$

The relevance of this will be discussed further later.

\subsubsection{The Spatiotemporal Commutator}

It is at this point that a commutation operator will be defined:

$$
\tilde{K}_{i}=\left[\partial_{t}, \partial_{i}\right]=\partial_{t} \partial_{i}-\partial_{i} \partial_{t}
$$


As in the static case, a postulate is made about the coordinate space: Even if the coordinate bases vectors are in motion (which implies a non-zero BetaSymbol), since the coordinates themselves are not dependent on time, the commutation operator should hold for all tensors [6]:

$$
\tilde{K}_{i} \mathcal{T}=0 \text {, where } \mathcal{T} \text { is a tensor of arbitrary rank. }
$$

This statement says more about the structure of the geometric coordinate space as it does about the Tensor Calculus, and can easily be identified sideby-side the concept of partial derivatives with respect to spatial dimensions; though they are embedded as curves in space, partial derivatives in their directions should commute [6]:

$$
\left[\partial_{i}, \partial_{j}\right]=\partial_{i} \partial_{j}-\partial_{j} \partial_{i}=2 \partial_{[i} \partial_{j]}=0
$$

More so, the operator is the first - in a line of soon determined operators which respect the following property:

In a dynamic coordinate frame, operators must preserve tensors.

In other words, for an invariant tensor $\phi$, the action of the commutator on the tensor will yield the following result:

$$
\tilde{K}_{j^{\prime}} \phi=J_{j^{\prime}}^{j} \tilde{K}_{j} \phi
$$

This result though intuitive is in fact remarkable. It implies that given other Tensors, scalars can be formed pertaining to 3D ambient space solely from the likes of expressions such as:

$$
\tilde{A}=A^{i}\left(\tilde{K}_{i} \phi\right)
$$

The vector analogue of the commutation re-inforces that the operator is an invariant vector operator all on its own:

$$
\tilde{\mathbf{K}} \phi=\mathbf{Z}^{i} \tilde{K}_{i} \phi \rightarrow \tilde{\mathbf{K}}^{\prime}=\tilde{\mathbf{K}}
$$

\subsubsection{Beta-Christoffel Relation}

The Spatiotemporal Commutator $\tilde{K}_{i}$, can be applied to the Basis (because the basis is a tensor by definition, as is the operator). This unifies the BetaSymbols \& Christoffel Symbols:

$$
\tilde{K}_{j} \mathbf{Z}_{i}=\partial_{t} \partial_{j} \mathbf{Z}_{i}-\partial_{j} \partial_{t} \mathbf{Z}_{i} \rightarrow \tilde{K}_{j} \mathbf{Z}_{i}=\partial_{t}\left(\Gamma_{i j}^{k} \mathbf{Z}_{k}\right)-\partial_{j}\left(\beta_{i}^{k} \mathbf{Z}_{k}\right)
$$


This equation can be simplified into the desired relationship:

$$
\mathbf{Z}^{k} \cdot \tilde{K}_{j} \mathbf{Z}_{i}=\partial_{t} \Gamma_{i j}^{k}-\partial_{j} \beta_{i}^{k}-\beta_{i}^{\ell} \Gamma_{j \ell}^{k}+\beta_{\ell}^{k} \Gamma_{i j}^{\ell}
$$

Since the Spatiotemporal Commutator should vanish on a tensor, the following relation is obtained:

$$
\partial_{t} \Gamma_{i j}^{k}=\partial_{j} \beta_{i}^{k}+\beta_{i}^{\ell} \Gamma_{j \ell}^{k}-\beta_{\ell}^{k} \Gamma_{i j}^{\ell}
$$

It is noteworthy to state that normally, one would be able to abbreviate the last three terms into a covariant derivative. This however is not possible since the Beta-Symbol does not transform as a tensor under a general dynamic transformation.

Finally, since the Christoffel Symbols are symmetric, and vanishes on its antisymmetric component $[1,2,3,4]$, the following antisymmetric identity is stated:

$$
\partial_{t} \Gamma_{[i j]}^{k}=0 \rightarrow \partial_{[j} \beta_{i]}^{k}=\Gamma_{\ell[i}^{k} \beta_{j]}^{\ell}
$$

This is an unmistakeable relation connecting the Christoffel Symbols to the Beta Symbols.

\subsubsection{Spatiotemporal Change of Coordinates}

The effects of changing the coordinate frame contain rules for transformation. In a new coordinate frame, the central heart of the topic is that the change of coordinates, unlike in Classical Tensor Calculus, depends on the temporal parameter [1]. This is expressed as:

$$
Z^{i} \rightarrow Z^{i}\left(t, Z^{\prime}\right) \quad, \quad Z^{i^{\prime}} \rightarrow Z^{i^{\prime}}(t, Z)
$$

This time dependence of the coordinate change is one of the issues which causes most of the complications in time derivatives. The variance in the coordinate change can be captured by two symbols. First, any curvilinear variance is captured by the standard Jacobian $[1,3]$ :

$$
\frac{\partial Z^{i}\left(t, Z^{\prime}\right)}{\partial Z^{i^{\prime}}}=J_{i^{\prime}}^{i}, \quad \frac{\partial Z^{i^{\prime}}(t, Z)}{\partial Z^{i}}=J_{i}^{i^{\prime}}
$$

In some cases, second order jacobians find application [1]:

$$
\frac{\partial^{2} Z^{i}}{\partial Z^{j^{\prime}} \partial Z^{i^{\prime}}}=J_{i^{\prime} j^{\prime}}^{i}, \frac{\partial^{2} Z^{i^{\prime}}}{\partial Z^{j} \partial Z^{i}}=J_{i j}^{i^{\prime}}
$$


Their definition allows for a special way to express the following identity:

$$
\partial_{k}\left(J_{i^{\prime}}^{i} J_{j}^{i^{\prime}}\right)=\partial_{k} \delta_{j}^{i}=0 \rightarrow J_{j}^{j^{\prime}} J_{k}^{k^{\prime}} J_{j^{\prime} k^{\prime}}^{i}+J_{i^{\prime}}^{i} J_{j k}^{i^{\prime}}=0
$$

And also allow for the definition of the transformation of the Christoffel Symbols [1]:

$$
\Gamma_{j^{\prime} k^{\prime}}^{i^{\prime}}=J_{i}^{i^{\prime}} J_{j^{\prime}}^{j} J_{k^{\prime}}^{k} \Gamma_{j k}^{i}+J_{i}^{i^{\prime}} J_{j^{\prime} k^{\prime}}^{i}
$$

Temporal variance in this new coordinate change can also be captured by the following Time jacobian [5]:

$$
J_{t}^{i}=\frac{\partial Z^{i}\left(t, Z^{\prime}\right)}{\partial t}, J_{t}^{i^{\prime}}=\frac{\partial Z^{i^{\prime}}(t, Z)}{\partial t}
$$

These Time jacobians can also be extended to define mixed jacobians [5]:

$$
J_{i^{\prime} t}^{i}=\frac{\partial^{2} Z^{i}}{\partial t \partial Z^{i^{\prime}}}, \quad J_{i t}^{i^{\prime}}=\frac{\partial Z^{i^{\prime}}}{\partial t \partial Z^{i}}
$$

This can be used to derive the very important identity by taking the time derivative of the Kronecker Delta [1]:

$$
\partial_{t}\left(J_{i^{\prime}}^{i} J_{j}^{i^{\prime}}\right)=\partial_{t} \delta_{j}^{i}=0 \rightarrow J_{i^{\prime} t}^{i} J_{j}^{i^{\prime}}+J_{i^{\prime}}^{i} J_{j t}^{i^{\prime}}=0
$$

With all these, the transformation for a partial time derivative of an invariant can be easily obtained. In addition, these can be used to show how the betasymbol transforms in a quasi-tensorial method:

$$
\beta_{j^{\prime}}^{i^{\prime}}=J_{i}^{i^{\prime}} J_{j^{\prime}}^{j} \beta_{j}^{i}+J_{i}^{i^{\prime}} J_{j^{\prime} t}^{i}+J_{i}^{i^{\prime}} J_{j^{\prime}}^{j} J_{t}^{k} \Gamma_{j k}^{i}
$$

This confirms the notion that the Beta-Symbol does not transform as a tensor, but much like the Christoffel Symbols, will be indispensable in defining a Tensorially invariant version of the Time Derivative of a Tensor.

\subsection{Transformation of a Partial Time Derivative: Is- sues Arise}

Suppose for simplicity, the Invariant Field, $\phi(t, Z)$ which is defined at every point in a continua/coordinate space $[1,6]$. Its time derivative is given by:

$$
\partial_{t} \phi=\partial_{t} \phi(t, Z)
$$

Under a change of coordinate, $\phi^{\prime}=\phi\left(t, Z^{\prime}\right)=\phi\left(t, Z\left(t, Z^{\prime}\right)\right)$. This unique dependence on the coordinates results in the following transformation:

$$
\partial_{t} \phi^{\prime}=\partial_{t} \phi\left(t, Z\left(t, Z^{\prime}\right)\right)=\partial_{t} \phi+\frac{\partial Z^{i}\left(t, Z^{\prime}\right)}{\partial t} \partial_{i} \phi
$$


This can be simplified into the following Tranformation Law [6]:

$$
\partial_{t} \phi^{\prime}=\left(\partial_{t}+J_{t}^{i} \nabla_{i}\right) \phi
$$

This presents issues when discussing that the field remains a tensor. Being a tensor of rank $(0,0)$ (an invariant) and since the time parameter is not a coordinate, under a change of coordinates it should remain an invariant! [1], but what is seen is a very different result. If the tensorial property does not hold for invariants, it can be easily proved that it does not hold for tensors of arbitrary rank. For a tensor of rank $(1,0)$, the transformation law is as follows:

$$
\partial_{t} \phi^{i^{\prime}}=J_{i}^{i^{\prime}}\left(\partial_{t} \phi^{i}+J_{t}^{j} \partial_{j} \phi^{i}\right)+J_{i t}^{i^{\prime}} \phi^{i}
$$

Where as for a tensor of rank $(0,1)$, the transformation law is as follows:

$$
\partial_{t} \phi_{i^{\prime}}=J_{i^{\prime}}^{i}\left(\partial_{t} \phi_{i}+J_{t}^{j} \partial_{j} \phi_{i}\right)+J_{i^{\prime} t}^{i} \phi_{i}
$$

The immediate implication of theses results is stunning. This implies that in laws such as in Eq.(1) and Eq.(2) [6, 7], the tensorial property for the fields under temporal analysis is not preserved under coordinate systems which are varying due to spatiotemporal distorting effects. This loss of tensorial invariance among invariants, and the extrapolation to tensors of higher rank automatically demands the development of a new time derivative operator which preserves certain symmetries in the Fields under Temporal Study, and this is the motivation of the following paper.

\subsection{A Similar Issue: The Calculus of Moving Surfaces}

\subsubsection{Geometry of CMS}

In CMS, a similar problem is encountered. Let $\psi(t, S)$ be an invariant field where $S=\left(S^{1}, S^{2}, \ldots, S^{\alpha}\right)$ refer to the Surface Coordinates of an arbitrary manifold. Here, the surface coordinates are related to the ambient space by the parametrization of a moving surface [1]:

$$
\mathbf{R}=\mathbf{R}(t, S)
$$

\subsubsection{Transformation of an Surface Invariant}

The surface coordinates under a change of coordinates posess the spatial Jacobians $J_{\alpha^{\prime}}^{\alpha} \& J_{\alpha}^{\alpha^{\prime}}$, and the temporal jacobian, $J_{t}^{\alpha}[1]$. For such a field when it transforms generally, this involves a change in Ambient Coordinates (a non-time-dependent transformation) and a change in Surface Coordinates 
(a time-dependent transformation). The invariant field transforms under time differentiation as such [1]:

$$
\partial_{t} \psi^{\prime}=\partial_{t} \psi\left(t, S\left(t, S^{\prime}\right)\right)=\partial_{t} \psi+J_{t}^{\alpha} \nabla_{\alpha} \psi
$$

This obstacle regarding the invariant's non-invariance requires a new derivative to be defined which preserves the tensorial property for invariants and tensors. This is defined for invariants as [1]:

$$
\dot{\nabla} \psi=\left(\partial_{t}-V^{\alpha} \nabla_{\alpha}\right) \psi(t, S)
$$

Under a variance in ambient and dynamic surface coordinates, this operator preserves the invariant properties and can be extended to tensorial arguments.

\section{The Temporal Field Derivative}

\subsection{From CMS to Moving Coordinate Frames}

Motivated by the CMS example in Eq.(35), an operator for a spatiotemporal tensor field of rank $(0,0) \phi(t, Z)$, must be found which remains invariant under a general transformation. Typically when searching for such an operator, one assumes a general form for the operator, and then specifies the following form of the operator under conditions of invariance and agreeance with the algebra [?]. The Operator will be denoted as the Temporal Field Derivative (so named because it focuses on time differentiation and operates on Spatiotemporal Fields); motivated by the CMS example, the general form of the operator will be proposed as:

$$
\dot{\tilde{\nabla}} \phi=\left(\partial_{t}+\Lambda^{i} \nabla_{i}+\Omega\right) \phi
$$

Where $\Lambda^{i}$ is a general algebraic object which obeys Einstein Summation Convention [2] (it will be established that $\Lambda^{i}$ is not a tensor shortly) and an arbitrary invariant field, $\Omega=\Omega(t, Z)$. This operator has several interesting properties. For example:

- It belongs to the classification as a linear operator

- It obeys the additive distributive property $\dot{\tilde{\nabla}}(\phi+\psi)=\dot{\tilde{\nabla}} \phi+\dot{\tilde{\nabla}} \psi$

- It is associative for a constant multiplied to a field: $\dot{\tilde{\nabla}}(c \phi)=c \dot{\tilde{\nabla}} \phi$

- It does not obey the product rule: $\dot{\tilde{\nabla}}(\phi \psi) \neq \psi \dot{\tilde{\nabla}} \phi+\phi \dot{\tilde{\nabla}} \psi$ 
- It does not obey the chain rule: $\dot{\vec{\nabla}} f(\phi) \neq \frac{d f}{d \phi} \dot{\tilde{\nabla}} \phi$

- The last two notions are due to the field $\Omega$ and will become problematic later.

\subsection{Invariance Condition}

If the operator is required to be invariant under a change of coordinates from one dynamic coordinate system to another, the following requirement is prescribed out of necessity:

$$
\dot{\tilde{\nabla}} \phi^{\prime}=\dot{\tilde{\nabla}} \phi
$$

Analyzing how the operator changes under a change in coordinates:

$$
\dot{\tilde{\nabla}} \phi^{\prime}=\left(\partial_{t}+\left(J_{t}^{i}+\Lambda^{i^{\prime}} J_{i^{\prime}}^{i}\right) \nabla_{i}+\Omega\right) \phi
$$

If invariance is to be satisfied for a tensor of rank $(0,0)$, the $\Lambda^{i}$ object must satisfy the following transformation:

$$
\Lambda^{i}=\Lambda^{i^{\prime}} J_{i^{\prime}}^{i}+J_{t}^{i} \quad \text { or } \quad \Lambda^{i^{\prime}} J_{i^{\prime}}^{i}=\Lambda^{i}-J_{t}^{i}
$$

\subsubsection{The Lambda Object: Symmetries Arise}

By its transformation law, it can easily be seen that the Lambda Object is not a tensor, but combines to form a tensorial operator, interestingly much like the function of the Christoffel Symbols in a Covariant Derivative [1, 2, 3]. Perhaps the most interesting property about this object is that if it is decomposed into a tensorial component, $T^{i}$ and a non-tensorial component, $\lambda^{i}$, the non-tensorial component must transform identically to the Lambda Object, regardless to the choice of the tensorial component which vanishes in the transformation law. Thus, the Lambda Object contains a transformation which preserves the tensorial invariance of the Temporal Field Derivative

$$
\Lambda^{i} \rightarrow \Lambda^{i}+T^{i}
$$

Where $T^{i}$ transforms like a tensor $\left(T^{i^{\prime}}=J_{i}^{i^{\prime}} T^{i}\right)$.

\subsubsection{A General Class of Operators}

The above Lambda Object's preservation of Tensorial Invaraince under a transformation ultimately also implies the Temporal Field Derivative should remain an invariant operator under the above transformation:

$$
\dot{\tilde{\nabla}} \rightarrow \dot{\tilde{\nabla}}+T^{i} \nabla_{i}
$$


This makes sense: if the Temporal Field Derivative transforms as a Tensor, then by the additive property of tensors, so will any linear combination of a tensor added to the operator. The second term of Eq.(40) is a directional derivative of $\phi$ in the direction of the tensor, $\vec{T}=T^{i} \mathbf{Z}_{i}$ and is thus a tensor [1].

\subsection{The Directed Temporal Field Derivative}

It can be seen that the original definition of the Temporal Field Derivative in Eq.(36) is the operator which is fixed by choosing the $\vec{T}$ tensor to be the zero vector $\mathbf{0}$. In full generality, the unique operator which preserves the tensorial property on a rank zero tensor is of the following form:

$$
\dot{\tilde{\nabla}}_{[\vec{T}]}=\partial_{t}+\left(\Lambda^{i}+T^{i}\right) \nabla_{i}+\Omega
$$

Thus, the Temporal Field Derivative (as far as its property to preserve tensors goes), admits a general class of operators, indexed by the choice of $T^{i}$ :

$$
\dot{\tilde{\nabla}}_{[\vec{T}]}=\dot{\tilde{\nabla}}_{[\overrightarrow{0}]}+\nabla_{\vec{T}}
$$

Where $\nabla_{\vec{T}}$ is defined as the directional derivative by 'citeCMS:

$$
\nabla_{\vec{T}}=T^{i} \nabla_{i}
$$

\subsection{Extending the Derivative to Higher Rank Tensors}

Up until now, only the action of the Temporal Field Derivative on tensors of rank $(0,0)$ have been discussed. Suppose instead, a tensor of rank $(1,0)$ is considered:

$$
\vec{\phi}=\phi^{i} \mathbf{Z}_{i}
$$

Applying the definition of the operator in Eq.(41) to the form of the tensor $\vec{\phi}=\phi^{i} \mathbf{Z}_{i}$ using the product rule, and recalling the definition of the Beta Symbols in Eq.(5), the action of the Temporal Field Derivative is as follows:

$$
\dot{\tilde{\nabla}}_{[\vec{T}]} \vec{\phi}=\left(\partial_{t} \phi^{j}+\phi^{k} \beta_{k}^{j}+\left(\Lambda^{i}+T^{i}\right) \nabla_{i} \phi^{j}+\Omega \phi^{j}\right) \mathbf{Z}_{j}
$$

\subsubsection{Introducing the Product Rule for Metrilinicity}

Interestingly, the action of the operator in Eq.(44) can all be contained within the Parentheses. This implies that the action of the operator does not need 
to depend on the basis vectors: this is essential; if the operator obeys the product rule, then the unescapable conclusion is reached that [1]:

The Product Rule imposed on the operator implies that the operator vanishes when applied to the basis vectors of a dynamic coordinate frame (ie. $\dot{\tilde{\nabla}}_{[\vec{T}]} \mathbf{Z}_{i}=\mathbf{0}$ ).

This is an important metrilinic property $[1,4]$. The only way which the operator can become commutative is if and only if $\Omega=0$. In this case, the operator obeys the product rule, and establishes a fundamental result: much like the Covariant Derivative [1, 3], the operator satisfies:

$$
\dot{\tilde{\nabla}}_{[\vec{T}]} \mathbf{Z}_{i}=\mathbf{0}
$$

The Temporal Field Derivative from Eq.(41) now has a new form which is given by:

$$
\dot{\tilde{\nabla}}_{[\vec{T}]}=\partial_{t}+\left(\Lambda^{i}+T^{i}\right) \nabla_{i}
$$

Consequently, the operator now also obeys the Chain Rule:

$$
\dot{\tilde{\nabla}}_{[\vec{T}]} f(\phi)=\frac{d f}{d \phi} \dot{\tilde{\nabla}}_{[\vec{T}]} \phi
$$

Also, by the operator obeying the product rule, the Temporal Field Derivative can be defined on a contravariant tensor:

$$
\dot{\tilde{\nabla}}_{[\vec{T}]} \phi^{i}=\left(\partial_{t}+\left(\Lambda^{j}+T^{j}\right) \nabla_{j}\right) \phi^{i}+\beta_{j}^{i} \phi^{j}
$$

Using a similar reasoning, for the contravariant basis, and a covariant basis, the action of the Temporal Field Derivative is:

$$
\dot{\tilde{\nabla}}_{[\vec{T}]} \mathbf{Z}^{i}=0, \quad \dot{\tilde{\nabla}}_{[\vec{T}]} \phi_{i}=\left(\partial_{t}+\left(\Lambda^{j}+T^{j}\right) \nabla_{j}\right) \phi_{i}-\beta_{i}^{j} \phi_{j}
$$

\subsubsection{The General Mixed Temporal Field Derivative}

Thus the Temporal Field Derivative can be presented in full generality for a second order tensor of rank $(1,1)$ :

$$
\dot{\tilde{\nabla}}_{[\vec{T}]} \phi_{j}^{i}=\left(\partial_{t}+\left(\Lambda^{k}+T^{k}\right) \nabla_{k}\right) \phi_{j}^{i}+\beta_{k}^{i} \phi_{j}^{k}-\beta_{j}^{k} \phi_{k}^{i}
$$

\subsubsection{The Metrilinic Property}

If the action of the Temporal Field Derivative on the contravariant \& covariant bases vanishes and obeys the Product Rule, then it can be seen that $[1]$ :

$$
\dot{\tilde{\nabla}}_{[\vec{T}]} Z_{i j}=\dot{\tilde{\nabla}}_{[\vec{T}]} Z^{i j}=\dot{\tilde{\nabla}}_{[\vec{T}]} \delta_{j}^{i}=0
$$




\subsection{The Temporal Field Derivative's Tensorial Prop- erty}

Finally, extending the tensorial properties of the operator, for a tensor of arbitrary rank $(k, m)$ the action of the Temporal Field Derivative is as follows:

$$
\begin{gathered}
\dot{\tilde{\nabla}} F_{j_{1} j_{2} \ldots j_{m}}^{i_{1} i_{2} \ldots i_{k}}=\left(\partial_{t}+\left(\Lambda^{k}+T^{k}\right) \nabla_{k}\right) \\
F_{j_{1} j_{2} \ldots j_{m}}^{i_{1} i_{2} \ldots i_{k}}+\beta_{p}^{i_{1}} F_{j_{1} j_{2} \ldots j_{m}}^{p i_{2} \ldots i_{k}}+\ldots+\beta_{p}^{i_{k}} F_{j_{1} j_{2} \ldots j_{m}}^{i_{1} \ldots i_{k-1} p} \\
-\beta_{j_{1}}^{q} F_{q j_{2} \ldots j_{m}}^{i_{1} i_{2} \ldots i_{k}}-\ldots-\beta_{j_{m}}^{q} F_{j_{1} \ldots j_{m-1}}^{i_{1} i_{2} \ldots i_{k}}
\end{gathered}
$$

It can be explicitly shown that according to the transformation of the Dynamic Beta Symbols $\beta_{j}^{i}$ from Eq.(30), and the transformation of the Lambda Pseudotensor $\Lambda^{i}$ from Eq.(38), the operator therefore satisfies the following transformation laws for the first few tensor ranks:

$$
\begin{aligned}
& \dot{\tilde{\nabla}}_{[\vec{T}]} \phi^{\prime}=\dot{\bar{\nabla}}_{[\overrightarrow{T T}} \phi \\
& \dot{\vec{\nabla}}_{[\vec{T}]} \phi^{i^{\prime}}=J_{i}^{i^{\prime}} \dot{\bar{\nabla}}_{[\vec{T}]} \phi^{i} \\
& \dot{\tilde{\nabla}}_{[\vec{T}]} \phi_{i^{\prime}}=J_{i^{\prime}}^{i} \dot{\bar{\nabla}}_{[\vec{T}} \phi_{i} \\
& \dot{\tilde{\nabla}}_{[\vec{T}]} \phi_{j^{\prime}}^{i^{\prime}}=J_{j^{\prime}}^{j} i_{i}^{i^{\prime}} \dot{\tilde{\nabla}}_{[\vec{T}]} \phi_{j}^{i}
\end{aligned}
$$

In the following section, this will now be explicitly derived for a Contravariant Tensor; for brevity the non-directed case, $\dot{\tilde{\nabla}}_{[\overrightarrow{0}]}=\left.\dot{\tilde{\nabla}}_{[\vec{T}]}\right|_{\vec{T}=\mathbf{0}}$ will be chosen.

\subsection{Explicit Derivation for a Tensor of Rank $(1,0)$}

For a tensor of rank $(1,0)$, under a transformation, its transformation law is:

$$
\dot{\tilde{\nabla}}_{[\overrightarrow{0}]} \phi^{i^{\prime}}=\partial_{t} \phi^{i^{\prime}}+\Lambda^{j^{\prime}} \nabla_{j^{\prime}} \phi^{i^{\prime}}+\beta_{j^{\prime}}^{i^{\prime}} \phi^{j^{\prime}}
$$

By utilizing jacobians, and recalling the tensor preserving property of the Covariant Derivative, this can be rewritten as:

$$
\dot{\tilde{\nabla}}_{[0]} \phi^{i^{\prime}}=\partial_{t} \phi^{i^{\prime}}+J_{i}^{i^{\prime}} \Lambda^{j^{\prime}} J_{j^{\prime}}^{j} \nabla_{j} \phi^{i}+\beta_{j^{\prime}}^{i^{\prime}} J_{m}^{j^{\prime}} \phi^{m}
$$

Recalling from the previously established transformation laws from Eq.(30), Eq.(32), and Eq.(38), the method that each of these terms transform is:

$$
\begin{aligned}
\beta_{j^{\prime}}^{i^{\prime}} m_{m}^{j^{\prime}} & =J_{i}^{i^{\prime}} \beta_{m}^{i}+J_{m}^{j^{\prime}} J_{i}^{i^{\prime}} J_{j^{\prime} t}^{i}+J_{i}^{i^{\prime}} J_{t}^{k} \Gamma_{m k}^{i} \\
\partial_{t} \phi^{i^{\prime}} & =J_{i}^{i^{\prime}}\left(\partial_{t} \phi^{i}+J_{t}^{j} \partial_{j} \phi^{i}\right)+J_{i t}^{i^{\prime}} \phi^{i} \\
\Lambda^{j^{\prime}} J_{j^{\prime}}^{j} & =\Lambda^{j}-J_{t}^{j}
\end{aligned}
$$


Thus after substituting the transformation laws and collecting the three terms of the Temporal Field Derivative, the transformation can be simplified as:

$$
\dot{\tilde{\nabla}}_{[\overrightarrow{0}]} \phi^{i^{\prime}}=J_{i}^{i^{\prime}} \dot{\tilde{\nabla}} \phi^{i}+J_{i t}^{i^{\prime}} \phi^{i}+J_{i}^{i^{\prime}} J_{t}^{j}\left(\partial_{j} \phi^{i}-\nabla_{j} \phi^{i}\right)+\left(J_{m}^{j^{\prime}} J_{i}^{i^{\prime}} J_{j^{\prime} t}^{i}+J_{i}^{i^{\prime}} J_{t}^{k} \Gamma_{m k}^{i}\right) \phi^{m}
$$

The covariant derivative's extra terms in parentheses can be simplified to produce the Christoffel Symbols according to $\nabla_{j} \phi^{i}=\partial_{j} \phi^{i}+\Gamma_{j k}^{i} \phi^{k}$. Utilizing this identity, the transformation is simplified to:

$$
\dot{\tilde{\nabla}}_{[\overrightarrow{0}]} \phi^{i^{\prime}}=J_{i}^{i^{\prime}} \dot{\tilde{\nabla}} \phi^{i}+J_{i t}^{i^{\prime}} \phi^{i}-J_{i}^{i^{\prime}} J_{t}^{j} \Gamma_{j k}^{i} \phi^{k}+J_{i}^{i^{\prime}}\left(J_{m}^{j^{\prime}} J_{j^{\prime} t}^{i}+J_{t}^{k} \Gamma_{m k}^{i}\right) \phi^{m}
$$

The two Christoffel Symbol terms annihilate eachother, which then produces the final result of:

$$
\dot{\tilde{\nabla}}_{[\overrightarrow{0}]} \phi^{i^{\prime}}=J_{i}^{i^{\prime}} \dot{\tilde{\nabla}} \phi^{i}+\left(J_{m t}^{i^{\prime}}+J_{m}^{j^{\prime}} J_{i}^{i^{\prime}} J_{j^{\prime} t}^{i}\right) \phi^{m}
$$

Finally the identity obtained in Eq.(29) is utilized to finally obtain the fundamental result:

$$
\dot{\tilde{\nabla}}_{[\overrightarrow{0}]} \phi^{i^{\prime}}=J_{i}^{i^{\prime}} \dot{\tilde{\nabla}} \phi^{i}
$$

Therefore the Tensorial Field Derivative preserves the tensorial properties of its operands even under all dynamic variations in the coordinate by arbitrary sources.

\section{First Order Commutations}

The Temporal Field Derivative from Eq.(47) contains redundant degrees of freedom left over within the $\vec{T}$ vector from assuring that the operator must be tensorial. These degrees of freedom can be reduced based on satisfying criteria for allowing the commutations of various operators assumed to commute in Classical Physics.

\subsection{Temporal Field Derivative Commutations}

For example, the commutation of the Temporal Field Derivative $\dot{\tilde{\nabla}}_{[\vec{T}]}$, and the standard covariant derivative $\nabla_{i}$, over a scalar invariant field $\phi$, explicitly depend on the $\vec{T}$ Tensor. Specifying that this commutation should vanish as the following condition:

$$
\left[\dot{\tilde{\nabla}}_{[\vec{T}]}, \nabla_{i}\right]=\dot{\tilde{\nabla}}_{[\vec{T}]} \nabla_{i}-\nabla_{i} \dot{\tilde{\nabla}}_{[\vec{T}]}=0
$$


Ultimately will be seen to impose conditions on $\vec{T}$ which result in decreasing the degrees of freedom for the Temporal Field Derivative $\dot{\tilde{\nabla}}_{[\vec{T}]}$.

Before imposing Eq.(54), first a simplified expression for the commutation of the two operators $\left[\dot{\tilde{\nabla}}_{[\vec{T}]}, \nabla_{i}\right]$ must be derived. The commutation of the Temporal Field Derivative with the Covariant Derivative can be separated into commutations with respect to the Temporal Field Derivative's two tensorial operators as per Eq.(42):

$$
\left[\dot{\tilde{\nabla}}_{[\vec{T}]}, \nabla_{i}\right] \phi=\left[\dot{\tilde{\nabla}}_{[\overrightarrow{0}]}+\nabla_{\vec{T}}, \nabla_{i}\right] \phi=\left[\dot{\tilde{\nabla}}_{[\overrightarrow{0}]}, \nabla_{i}\right] \phi+\left[\nabla_{\vec{T}}, \nabla_{i}\right] \phi
$$

After expanding the terms, the commutation of the Directional Derivative with the Covariant Derivative can be seen to have the following simplification; the right side of the relation is a tensor, much like the left side of the equation is.

$$
\left[\nabla_{\vec{T}}, \nabla_{i}\right] \phi=-\left(\nabla_{i} T^{j}\right) \partial_{j} \phi
$$

As for the commutation of the Temporal Field Derivative (with $\vec{T}=\mathbf{0}$ ie. $\dot{\tilde{\nabla}}_{[\mathbf{0}]}$ ) and the Covariant Derivative $\nabla_{i}$ : after some work, the commutation can be resolved into the following form:

$$
\left[\dot{\tilde{\nabla}}_{[\overrightarrow{0}]}, \nabla_{i}\right] \phi=\tilde{K}_{i} \phi-\left(\partial_{i} \Lambda^{j}+\Gamma_{i k}^{j} \Lambda^{k}+\beta_{i}^{j}\right) \nabla_{j} \phi
$$

The terms within the parentheses can be abbreviated into a new symbol, denoted by $\Omega^{j}{ }_{i}$ :

$$
\Omega_{i}^{j}=\partial_{i} \Lambda^{j}+\Gamma_{i k}^{j} \Lambda^{k}+\beta_{i}^{j}
$$

Recalling that the Spatiotemporal Commutator, $\tilde{K}_{i}$ vanishes on an invariant (ie. $\tilde{K}_{i} \phi=0$ ), then the commutation is simplified as:

$$
\left[\dot{\tilde{\nabla}}_{[\overrightarrow{0}]}, \nabla_{i}\right] \phi=-\Omega^{j}{ }_{i} \partial_{j} \phi
$$

Thus, the ultimate commutation of the Temporal Field Derivative with the Covariant Derivative substituting Eq.(56) \& Eq.(58) into Eq.(55) is:

$$
\left[\dot{\tilde{\nabla}}_{[\vec{T}]}, \nabla_{i}\right] \phi=-\left(\Omega_{i}^{j}+\nabla_{i} T^{j}\right) \nabla_{j} \phi
$$

\subsection{Tensorial Identity of the Commutation}

The left side of the commutation is a tensor since it is the commutation of tensorial Operators: the Temporal Field Derivative $\dot{\tilde{\nabla}}_{[\vec{T}]} \&$ the Covarant Derivative $\nabla_{i}$. In order to verify that the right side of the equation behaves 
like a tensor too, the method by which the new Omega symbol from Eq.(57) transforms is required:

$$
\Omega_{i^{\prime}}^{j^{\prime}}=\partial_{i^{\prime}} \Lambda^{j^{\prime}}+\Gamma_{i^{\prime} k^{\prime}}^{j^{\prime}} \Lambda^{k^{\prime}}+\beta_{i^{\prime}}^{j^{\prime}}
$$

This can be obtained rapidly recalling Eq.(38):

$$
\Omega^{j^{\prime}}{ }_{i^{\prime}}=\partial_{i^{\prime}}\left(J_{j}^{j^{\prime}}\right)\left(\Lambda^{j}-J_{t}^{j}\right)+J_{j}^{j^{\prime}} \partial_{i^{\prime}}\left(\Lambda^{j}-J_{t}^{j}\right)+\Gamma_{i^{\prime} k^{\prime}}^{j^{\prime}} \Lambda^{k^{\prime}}+\beta_{i^{\prime}}^{j^{\prime}}
$$

After expanding and simplifying the Transformation then substituting Eq.(26), the following shows how the Omega tensor transforms:

$\Omega_{i^{\prime}}^{j^{\prime}}=J_{i^{\prime}}^{i} J_{i j}^{j^{\prime}}\left(\Lambda^{j}-J_{t}^{j}\right)+J_{j}^{j^{\prime}}\left(J_{i^{\prime}}^{i} \partial_{i} \Lambda^{j}-J_{i^{\prime} t}^{j}\right)+\left(J_{j}^{j^{\prime}} J_{i^{\prime}}^{i} \Gamma_{i m}^{j}+J_{j}^{j^{\prime}} J_{i^{\prime} k^{\prime}}^{j} J_{m}^{k^{\prime}}\right)\left(\Lambda^{m}-J_{t}^{m}\right)+\beta_{i^{\prime}}^{j^{\prime}}$

Using Eq.(30), expanding, simplifying, and grouping terms into the Omega symbol reduces the above transformation to:

$$
\Omega^{j^{\prime}}{ }_{i^{\prime}}=J_{j}^{j^{\prime}} J_{i^{\prime}}^{i} \Omega_{i}^{j}+\left(J_{i^{\prime}}^{i} J_{i m}^{j^{\prime}}+J_{j}^{j^{\prime}} J_{i^{\prime} k^{\prime}}^{j} J_{m}^{k^{\prime}}\right)\left(\Lambda^{m}-J_{t}^{m}\right)
$$

Finally, applying Eq.(25) for the terms in parentheses, all the terms vanish and the tensorial form re-appears:

$$
\Omega_{i^{\prime}}^{j^{\prime}}=J_{j}^{j^{\prime}} J_{i^{\prime}}^{i} \Omega_{i}^{j}
$$

\subsubsection{Verification of an earlier Tensorial Statement}

Astonishingly so, the Omega Symbol is a tensor as shown by Eq.(60), however; as much work demonstrates this, it in fact confirms a previously stated result. Since the form of the commutation on an invariant, in full generality assuming for argument that $\tilde{K}_{i} \phi \neq 0$, is:

$$
\left[\dot{\tilde{\nabla}}_{[\vec{T}]}, \nabla_{i}\right] \psi=\tilde{K}_{i} \psi-\left(\Omega^{j}{ }_{i}+\nabla_{i} T^{j}\right) \nabla_{j} \psi
$$

If the Omega Symbol is a tensor as established by Eq.(60), and the Covariant Derivative of the Directional Vector $\nabla_{j} T^{i}$ is a tensor, and since the left side of the above equation (ie. $\left[\dot{\tilde{\nabla}}_{[\vec{T}]}, \nabla_{i}\right] \psi$ ) is a tensor, therefore the Spatiotemporal Commutator $\tilde{K}_{i}$, must transform like tensor also! This is an elegant, and simple proof that the Spatiotemporal Commutator $\tilde{K}_{i}$, is a tensor as stated previously by Eq.(16). However, for a full proof, this can be easily derived by analyzing how the Spatiotemporal Commutator transforms:

$$
\tilde{K}_{i^{\prime}} \phi=\left[\partial_{t}, \partial_{i^{\prime}}\right] \phi^{\prime}=\partial_{t} \partial_{i^{\prime}} \phi-\partial_{i^{\prime}} \partial_{t} \phi^{\prime}
$$


Substituting in Eq.(31) and acknowledging that $\partial_{i^{\prime}} \phi=J_{i^{\prime}}^{i} \partial_{i} \phi$ the transformation relation can be expanded and simplified to the following,

$$
\tilde{K}_{i^{\prime}} \phi=J_{i^{\prime}}^{i}\left(\partial_{t} \partial_{i} \phi+J_{t}^{j} \partial_{j} \partial_{i} \phi\right)+J_{i^{\prime} t}^{i} \partial_{i} \phi-J_{i^{\prime}}^{i} \partial_{i}\left(\partial_{t} \phi+J_{t}^{j} \partial_{j} \phi\right)
$$

Rearranging the terms to re-form $\tilde{K}_{i}$ and collecting all remaining terms the transformation relation is reduced to,

$$
\tilde{K}_{i^{\prime}} \phi=J_{i^{\prime}}^{i} \tilde{K}_{i} \phi+J_{i^{\prime}}^{i} J_{t}^{j} \partial_{j} \partial_{i} \phi+J_{i^{\prime} t}^{i} \partial_{i} \phi-J_{i^{\prime} t}^{j} \partial_{j} \phi-J_{i^{\prime}}^{i} J_{t}^{j} \partial_{i} \partial_{j} \phi
$$

Aknowledging that $\partial_{[i} \partial_{j]}=0[6]$ the result assumes the tensorial form:

$$
\tilde{K}_{i^{\prime}} \phi=J_{i^{\prime}}^{i} \tilde{K}_{i} \phi+2 J_{i^{\prime}}^{i} J_{t}^{j} \partial_{[j} \partial_{i]} \phi=J_{i^{\prime}}^{i} \tilde{K}_{i} \phi
$$

Thus at present, there are two operators which preserve the Tensorial identity of the Tensorial arguments:

$$
\tilde{K}_{i^{\prime}} \phi=J_{i^{\prime}}^{i} \tilde{K}_{i} \phi, \quad \dot{\tilde{\nabla}}_{[\vec{T}]} \phi_{i^{\prime}}=J_{i^{\prime}}^{i} \dot{\tilde{\nabla}}_{[\vec{T}]} \phi_{i}
$$

\subsection{Commutation of the Tensorial Operator}

As stated earlier in Eq.(54), in agreeing with standard Classical Physics, the commutation of the Temporal Field Derivative with the Covariant Derivative may be desired to vanish.

$$
\left[\dot{\tilde{\nabla}}_{[\vec{T}]}, \nabla_{i}\right] \phi=0
$$

By identification with the simplification in Eq.(59), we see that the following identity must hold true:

$$
\left(\Omega^{j}{ }_{i}+\nabla_{i} T^{j}\right) \nabla_{j} \phi=0
$$

This condition should vanish for all continuous Spatiotemporal Fields $\phi$ (and by extension, their derivatives $\left.\nabla_{i} \phi\right)$ and thus, produces a Tensor Equation Condition stated succinctly as:

$$
\nabla_{i} T^{j}=-\Omega^{j}
$$

Since both sides of Eq.(62) are in fact tensors, the equation holds true in a dynamic coordinate frame where $\left.\beta^{i}\right) j \neq 0$. 


\subsubsection{Further Implications of the Condition}

The condition seen in Eq.(62) is an example of a tensor condition restricting the Free Parameters of the $\vec{T}$ vector. In fact, if both sides of this equation are multiplied with the Basis $\mathbf{Z}_{i}$, and since it is metrilinic with respect to the covariant derivative, the tensor equation can be abbreviated into an explicit vector equation:

$$
\partial_{i} \vec{T}=-\Omega^{j}{ }_{i} \mathbf{Z}_{j}
$$

After recalling the definition of the Omega symbol in Eq.(57) and simplifying the right side of the equation, the following condition forms. It can be seen to have a deep connection with the geometry and dynamics of the coordinate frame, but this connection will be made on a deeper level later:

$$
\partial_{i}(\vec{T}+\vec{\Lambda})=-\partial_{t} \mathbf{Z}_{i}
$$

As a last note, this condition will directly impact and restrict the form of the Temporal Field Derivative. Since its form on invariants recalling from Eq.(41) is:

$$
\dot{\tilde{\nabla}}_{[\vec{T}]}=\partial_{t}+\left(\Lambda^{i}+T^{i}\right) \nabla_{i}
$$

It will have a different form dictated by the condition in Eq.(62). This condition almost defines all three components of the Directing Vector, $\vec{T}$. Having rigorously examined the $\vec{T}$ vector, it is time the $\Lambda^{i}$ object is examined.

\section{Determining the Lambda Object}

It was earlier seen in Eq.(38) that the Lambda Algebraic Object clearly is not a tensor by its tranformation law dictated by the way that the Temporal Field Derivative demands invariant transformations:

$$
\Lambda^{i^{\prime}} J_{i^{\prime}}^{i}=\Lambda^{i}-J_{t}^{i}
$$

Determining the identity of an object which abides by a calculus that does not acknowledge the transformations of Tensors is no easy feat. The form of the $\Lambda^{i}$ tensor is obtained by exploring the geometry of the space surrounding the Dynamic Coordinate Frame and deriving, then combining objects which transform in a similar way:

\subsection{The Variance Speed}

The Position Vector, $\mathbf{R}$, of a particle is re-examined given a deeper understanding of the motion of the Coordinate Frame. Now, the Position Vector 
is realized to depend on time even if a particle is not in motion, but the coordinate frame is, $\mathbf{R}=\mathbf{R}(t, Z)$. The Position Vector's curvilinear variance pertaining to a position of a particle with coordinates $Z^{i}$ was adressed earlier in Eq.(4) and is given by the Basis vectors by the relation:

$$
\partial_{i} \mathbf{R}=\mathbf{Z}_{i}
$$

The Position Vector's temporal variance was not analyzed. For a particle given in coordinates by the position vector $\mathbf{R}$, if the coordinate frame is moving (assuming the particle is stationary) then it still has a variance denoted by the Variance Speed given as:

$$
\partial_{t} \mathbf{R}=\vec{Y}
$$

Under a change of coordinate frames, the variance speed can be analyzed on how it changes. Assuming that the position Vector contains the form $\mathbf{R}=\mathbf{R}(t, Z)$, under a change in coordinate system, it will change as $\mathbf{R}^{\prime}=$ $\mathbf{R}\left(t, Z\left(t, Z^{\prime}\right)\right.$. After differentiating it with respect to time, the speed is observed to change according to the following:

$$
(\vec{Y})^{\prime}=\vec{Y}+J_{t}^{i} \mathbf{Z}_{i}
$$

This is a manner of saying:

The Variance Speed in a different frame, is the original Variance Speed plus the Temporal Variance in the new coordinate system projected along the Original System's Basis Vectors.

This is the analogue of the elementary addition rule for vectors. This variance speed is of crucial importance to the concept of the Temporal Field Derivative as it will be seen soon. This is one object which completely determines the change in the coordinate changes.

\subsubsection{The Variance Speed-Beta Symbol's Relationship}

Supposing the Variance Speed can be put into a component-by-component form:

$$
\vec{Y}=Y^{i} \mathbf{Z}_{i}
$$

This definition implies a very fundamental identity. Since $\tilde{K}_{i}=0$ and due to the metrilinicity of the bases with respect to the Temporal Field Derivative, $\dot{\tilde{\nabla}}_{[\vec{T}]} \mathbf{Z}_{i}=\mathbf{0}$, then:

$$
\partial_{i}\left(\partial_{t} \mathbf{R}\right)=\partial_{t}\left(\partial_{i} \mathbf{R}\right) \rightarrow \partial_{i} \vec{Y}=\partial_{t} \mathbf{Z}_{i}=\beta_{i}^{j} \mathbf{Z}_{j}
$$


Thus, the following relation is obtained:

$$
\beta_{i}^{j}=\partial_{i} Y^{j}+Y^{k} \Gamma_{i k}^{j}
$$

Using the earlier identity established in Eq.(20), this fundamental identity is obtained:

$$
\partial_{t} \Gamma_{i j}^{k}=Y^{\ell} R_{i j \ell}^{k}+\partial_{j} \partial_{i} Y^{k}+2 \Gamma_{\ell(j}^{k} \partial_{i)} Y^{\ell}+\Gamma_{i j, \ell}^{k} Y^{\ell}-\Gamma_{i j}^{\ell} \partial_{\ell} Y^{k}
$$

Where the Riemann Curvature Tensor shows usage here. By taking the antisymmetric component of the equation, many of the terms vanish due to their symmetric nature and the following identity lends itself:

$$
Y^{\ell} R_{[i j] \ell}^{k}=0
$$

\subsection{Guessing a Form: The Variance Speed Form}

Now that the Variance Speed is explored and is established to not transform as a tensor outlined in Eq.(65), the questions remains if it can be used in some combination as a substitute for the Lambda object $\Lambda^{i}$. By substituting Eq.(67) into Eq.(65), the following identity results:

$$
\left(Y^{i^{\prime}} J_{i^{\prime}}^{i}-Y^{i}-J_{t}^{i}\right) \mathbf{Z}_{i}=0
$$

By the correspondence $\Lambda^{i}=-Y^{i}$, Eq.(72) is satisfied and $\left(-Y^{i}\right)$ is an algebraic object which transforms precicely in the manner required for the Invariance promised by the Temporal Field Derivative from Eq.(38). This is sufficient to give a more exact form for the Temporal Field Derivative denoted as:

$$
\dot{\tilde{\nabla}}_{[\vec{T}]}^{(\vec{Y})}=\partial_{t}+\left(T^{i}-Y^{i}\right) \nabla_{i}
$$

This form of the operator is seen to transform invariantly even more obviously than before according to the elementary rule for the addition of tensors \& vectors:

$$
\begin{aligned}
& \dot{\tilde{\nabla}}_{[\vec{T}]}^{(\vec{Y})} \phi^{\prime}=\partial_{t} \phi^{\prime}+\left(T^{i^{\prime}}-Y^{i^{\prime}}\right) \partial_{i^{\prime}} \phi \\
& \dot{\tilde{\nabla}}_{[\vec{Y}]}^{(\vec{Y})} \phi^{\prime}=\partial_{t} \phi+J_{t}^{i} \partial_{i} \phi+\left(J_{i^{\prime}}^{i} T^{i^{\prime}}-J_{i^{\prime}}^{i} Y^{i^{\prime}}\right) \partial_{i} \phi \\
& \dot{\tilde{\nabla}}_{[\vec{Y})}^{(\vec{T})} \phi^{\prime}=\partial_{t} \phi+J_{t}^{i} \partial_{i} \phi+\left(T^{i}-Y^{i}-J_{t}^{i}\right) \partial_{i} \phi \\
& \dot{\tilde{\nabla}}_{[\vec{Y}]}^{(\vec{Y})} \phi^{\prime}=\partial_{t} \phi+\left(T^{i}-Y^{i}\right) \partial_{i} \phi+J_{t}^{i} \partial_{i} \phi-J_{t}^{i} \partial_{i} \phi
\end{aligned}
$$


To obtain the final expected tensorial transformation law:

$$
\dot{\tilde{\nabla}}_{[\vec{T}]}^{(\vec{Y})} \phi^{\prime}=\dot{\tilde{\nabla}}_{[\vec{T}]}^{(\vec{Y})} \phi
$$

If the spatiotemporal field $\phi$, does not alter in time (ie. just a spatial field $\phi=$ $\phi(Z)$ ), then the Temporal Field Derivative Reduces to the scaled Directional Derivative in the direction of the $\vec{T}$ vector and the Variance Speed (which is an intuitive result since the derivative corrects for the dynamic coordinate system's motion):

$$
\dot{\tilde{\nabla}}_{[\vec{T}]}^{(\vec{Y})} \phi(Z)=\nabla_{(\vec{T}-\vec{Y})} \phi
$$

In addition (and perhaps most importantly), for any spatiotemporal field, if the coordinate frame is not moving at all (or negligibly) then the operator will reduce down to a partial derivative assuming the $\vec{T}$ is fixed at the zero vector, $\vec{T}=0$ :

$$
\left.\dot{\tilde{\nabla}}_{[\overrightarrow{0}]}^{(\vec{Y})} \phi\right|_{|\vec{Y}| \approx 0} \approx \partial_{t} \phi
$$

This is the motivation of the paper and the motivation of the application of the operator: under low variance speeds (when the coordinate frame is barely moving $\vec{Y} \approx 0$ ), the operator makes the time derivative of an invariant spatiotemporal field approximately invariant. In addition, the following form of the Temporal Field Derivative is also defined:

$$
\dot{\tilde{\nabla}}^{(\vec{Y})}=\dot{\tilde{\nabla}}_{[\overrightarrow{0}]}^{(\vec{Y})}
$$

By this, the familiar identity from Eq.(42) reappears:

$$
\dot{\tilde{\nabla}}_{[\vec{T}]}^{(\vec{Y})}=\dot{\tilde{\nabla}}^{(\vec{Y})}+\nabla_{\vec{T}}
$$

The Variance Speed can be identified with the dynamic effects due to a variety of scenarios:

- Effects of General Relativity during the movement of a nearby Black Hole, during the process of Gravitational Waves, or due to a dynamic change of the Stress and Energy Tensor $[8,7]$

- A coodinate system pulsation due to its overlay on the cross-section of a pulsating artery [10]

- A dynamic 3D coordinate system capturing the internal effects of cellular processes [11]. 


\subsection{The Variance Speed \& First Order Commutations}

One of the most interesting applications of the Variance Speed is its relation to the commutation of the Temporal Field Derivative and the Covariant Derivative from earlier.

Recalling the relationship the Variance Speed holds to the Beta Symbol in Eq.(68):

$$
\partial_{i} \vec{Y}-\partial_{t} \mathbf{Z}_{i}=\mathbf{0}
$$

This can be used to re-affirm Eq.(62) and also re-affirm the definition of the Temporal Field Derivative from Eq.(73). Recalling from Eq.(63), the the two operators commute when the following condition is satisfied:

$$
\partial_{i}(\vec{T}+\vec{\Lambda})=-\partial_{t} \mathbf{Z}_{i}
$$

Using the identity in Eq.(68), this can be developed further:

$$
\partial_{i}(\vec{T}+\vec{\Lambda}+\vec{Y})=\mathbf{0}
$$

Recalling $\vec{\Lambda}=-\vec{Y}$ from Eq.(73), the following identity holds in vector form and in tensor component form:

$$
\partial_{j} \vec{T}=\mathbf{0} \quad \text { or } \quad \nabla_{j} T^{i}=0
$$

This condition seems contradictory to the earlier condition from Eq.(62) which required the following:

$$
\nabla_{i} T^{j}=-\Omega^{j}{ }_{i}
$$

As it implies that $\Omega^{j}{ }_{i}=0$; however this definition is in fact one in the same! Recalling that the definition of the Omega tensor in Eq.(57) as:

$$
\Omega_{i}^{j}=\partial_{i} \Lambda^{j}+\Gamma_{i k}^{j} \Lambda^{k}+\beta_{i}^{j}
$$

And recalling from the earlier transformation law for the Variance Speed Eq.(72), assuming that $\Lambda^{i}=-Y^{i}$ does indeed produce the result:

$$
\Omega^{i}{ }_{j}=0
$$

Confirming the earlier implications from Eq.(62) \& Eq.(78), and solidifying the definition of $\Lambda^{i}$ as $\left(-Y^{i}\right)$. So then, the Temporal Field Derivative will update its definition as well:

$$
\dot{\tilde{\nabla}}_{[\vec{T}]} \Rightarrow\left\{\dot{\tilde{\nabla}}_{[\vec{T}]}^{(\vec{Y})}, \quad \partial_{i} \vec{T}=0\right\}
$$


Therefore all this outlines a final form for the Temporal Field Derivative on Invariants and Tensors of Higher orders. For an invariant (ie. tensor of rank $(0,0))$, the Temporal Field Derivative's developed definition is as follows:

$$
\dot{\tilde{\nabla}}_{[\vec{T}]}^{(\vec{Y})} \phi=\partial_{t} \phi+\left(T^{i}-Y^{i}\right) \partial_{i} \phi \quad, \quad \nabla_{j} T^{i}=0
$$

This can be used with full confidence in applying it to models within Dynamic Coodinate Frames being distorted in time

\section{$5 \quad$ Introducing Notions of Spatio-Temporality}

Until now, the framework proposed preserves Spatiotemporal Fields in a tensorial way when coordinates are changed from one system to another under a dynamic continuous distortion. The framework has drawbacks when considering the concept that in other prominent Physics Theories such as General Relativity, Space and Time are unified into an complete 4D spacetime realm $[7]$.

WIth the current framework proposed in this paper, the transformations from cordinate space to coordinate space depend explicitly on time because the 3 Spatial Dimensions are connected to the time coordinate via a non-linear relation $Z^{i^{\prime}}=Z^{i^{\prime}}(t, Z)$. Thus, the $3 \mathrm{D}+1 \mathrm{D}$ space that is referred to in General Relativity deserves a special treatment when being referred to by this framework. A theoretical method for doing so wil be proposed.

\subsection{Geometry of the 4D Space}

Under the spatial constructions above, all spatiotemporal fields can be parametrized through the use of the following field notation:

$$
\psi=\psi(\zeta)
$$

Where here, $\zeta$ is an abbreviated form of the 4-coordinate space composed of: $\left(t, Z^{1}, Z^{2}, Z^{3}\right)$. In component form, indivicual coordinates will be referred to by $\zeta^{\sigma}$; also, greek indices will continuously be utilized to indicate a $4 \mathrm{D}$ space and roman indices to indicate the 3D Ambient Space. These coordinates can 
be separated from a 4D space, into a $1 \mathrm{D}$ and $3 \mathrm{D}$ coordinate space:

$$
\zeta^{\sigma}=\left(\begin{array}{c}
\zeta^{0} \\
\zeta^{1} \\
\zeta^{2} \\
\zeta^{3}
\end{array}\right)=\left(\begin{array}{c}
t \\
Z^{1} \\
Z^{2} \\
Z^{3}
\end{array}\right)
$$

For brevity, this can be abbreviated into:

$$
\zeta^{\sigma}=\left(\begin{array}{c}
\zeta^{0} \\
\zeta^{i}
\end{array}\right)=\left(\begin{array}{c}
t \\
Z^{i}
\end{array}\right)
$$

This parentheses notation will be used often to denote the $1 \mathrm{D} \& 3 \mathrm{D}$ components of a 4D Tensor Object where $\sigma=0$ refers to the time components. Both the Temporal Field Derivative from Eq.(81) \& the Covariant Derivative $\nabla_{i}$ tensorial operators can be combined into one superior Spatiotemporal Field Derivative. This is useful for defining differential connections in the $4 \mathrm{D}$ space:

$$
\ddot{\nabla}_{\sigma}^{\{\vec{T}\}}=\left(\begin{array}{c}
\dot{\tilde{\nabla}}_{[\vec{Y}]}^{(\vec{Y})} \\
\nabla_{i}
\end{array}\right) \text {, where } \nabla_{i} T^{j}=0
$$

\subsection{Vanishing of the Invariant Temporal Commutation}

It is well established that for covariant derivatives acting on an invariant field, the following identity holds $[1,6]$ :

$$
\left[\nabla_{i}, \nabla_{j}\right] \phi=\left[\partial_{i}, \partial_{j}\right] \phi=0
$$

It has already been established that the Temporal Field Derivative is one way to incorporate temporal invariance into a dynamic coordinate system as outlined by Eq.(74). The Covariant Derivatives fascilitate the same objective of incorporating curvilinear tensorial invariance in a dynamic coordinate system $\{$ reference $\}$. If the two invariant differential operators $\dot{\tilde{\nabla}}_{[\vec{T}]}^{(\vec{Y})}$ and $\nabla_{i}$ are combined into a third operator such as the Spatiotemporal Field Derivative $\ddot{\nabla}_{\sigma}^{\{\vec{T}\}}$, a set of operators which transform as a tensors is obtained. Much like the 3D coordinate system analogues stated in Eq.(54) \& Eq.(85), the following commutation condition will be imposed:

$$
\left[\ddot{\nabla}_{\sigma}^{\{\vec{T}\}}, \ddot{\nabla}_{\lambda}^{\{\vec{U}\}}\right] \phi=0
$$

Two different Directing Tensors, $\vec{T}$ and $\vec{U}$, are chosen to preserve full generality with the superimposed condition that $\nabla_{j} T^{i}=\nabla_{j} U^{i}=0$. Expanding 
the definition of the Spatiotemporal Field Derivative, Eq.(86) has four cases:

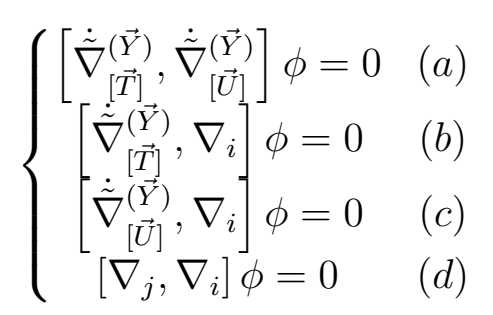

Where Eq.(b) \& Eq.(c) are essentially the same condition imposed on $\vec{T}$ and $\vec{U}$. Eq.(d) is automatically satisfied based on the Raw Structure of the Connection in 3D Space, and the principle that partial derivatives commute in the Ambient 3D Space. The remaining three equations can in fact be all satisfied simultaneously. It has been previosly that if the Temporal Field Derivative is chosen to be $\dot{\tilde{\nabla}}_{[\vec{T}]}^{(\vec{Y})}$, for any vector $\vec{T}$ such that $\nabla_{j} T^{i}=0$, then Eq. (b) and Eq.(c) are simultaneously satisfied as stated by Eq.(78). As a minor note used later, if two vectors' covariant derivatives vanish, then necessarily:

$$
\mathcal{L}_{\vec{T}} \vec{U}=-\mathcal{L}_{\vec{U}} \vec{T}=\overrightarrow{0}
$$

Where, $\mathcal{L}_{\vec{A}} \vec{B}=2 \mathbf{Z}_{j} A^{[i} \nabla_{i} B^{j]}$ is the Lie derivative of the system. Thus, only Eq.(a) must still be satisfied:

$$
\left[\dot{\tilde{\nabla}}_{[\vec{T}]}^{(\vec{Y})}, \dot{\tilde{\nabla}}_{[\vec{U}]}^{(\vec{Y})}\right] \phi=0
$$

After expanding the commutation according to the Temporal Field Derivative's decomposition as a Temporal Field Derivative with $\vec{T}=0$, and a Directional Derivative in the direction of $\vec{T}$ from Eq.(42),

$$
\left[\dot{\tilde{\nabla}}_{[\vec{T}]}, \dot{\tilde{\nabla}}_{[\vec{U}]}\right] \phi=\left[\dot{\tilde{\nabla}}^{(\vec{Y})}+\nabla_{\vec{T}}, \dot{\tilde{\nabla}}^{(\vec{Y})}+\nabla_{\vec{U}}\right] \phi
$$

This commutation can be split into many smaller commutations:

$$
\left[\dot{\tilde{\nabla}}_{[\vec{T}]}, \dot{\tilde{\nabla}}_{[\vec{U}]}\right] \phi=\left[\dot{\tilde{\nabla}}^{(\vec{Y})}, \dot{\tilde{\nabla}}^{(\vec{Y})}\right] \phi+\left[\dot{\tilde{\nabla}}^{(\vec{Y})}, \nabla_{\vec{U}}\right] \phi+\left[\nabla_{\vec{T}}, \dot{\tilde{\nabla}}^{(\vec{Y})}\right] \phi+\left[\nabla_{\vec{T}}, \nabla_{\vec{U}}\right] \phi
$$

Noticing the first commutation vanishes (by virtue of commuting identitical operators), the commutations can be re-arranged into the following:

$$
\left[\dot{\tilde{\nabla}}_{[\vec{T}]}, \dot{\tilde{\nabla}}_{[\vec{U}]}\right] \phi=\left[\dot{\tilde{\nabla}}^{(\vec{Y})}, \nabla_{\vec{U}}\right]-\left[\dot{\tilde{\nabla}}^{(\vec{Y})}, \nabla_{\vec{T}}\right] \phi+\left[\nabla_{\vec{T}}, \nabla_{\vec{U}}\right] \phi=\left[\dot{\tilde{\nabla}}^{(\vec{Y})}, \nabla_{\vec{U}}-\nabla_{\vec{T}}\right] \phi+\left[\nabla_{\vec{T}}, \nabla_{\vec{U}}\right] \phi
$$

And thus the commutation can be further simplified to a cleaner form:

$$
\left[\dot{\tilde{\nabla}}_{[\vec{T}]}, \dot{\tilde{\nabla}}_{[\vec{U}]}\right] \phi=\left[\dot{\tilde{\nabla}}^{(\vec{Y})}, \nabla_{\vec{U}-\vec{T}}\right] \phi+\left[\nabla_{\vec{T}}, \nabla_{\vec{U}}\right] \phi
$$


Defining a new vector, $\vec{C}=\vec{U}-\vec{T}$ for a cleaner appearance of the derivation, the simplification can be continued:

$$
\left[\dot{\tilde{\nabla}}_{[\vec{T}]}, \dot{\tilde{\nabla}}_{[\vec{U}]}\right] \phi=\left[\dot{\tilde{\nabla}}^{(\vec{Y})}, \nabla_{\vec{C}}\right] \phi+\left[\nabla_{\vec{T}}, \nabla_{\vec{U}}\right] \phi
$$

So ultimately after expanding the commutation, two commutations are obtained as the result. The second commutation reduces to the simplification:

$$
\left[\nabla_{\vec{T}}, \nabla_{\vec{U}}\right] \phi=\left(\mathcal{L}_{\vec{U}} \vec{T}\right) \cdot \vec{\nabla} \phi=0
$$

Where $\vec{\nabla}$ is defined as $\vec{\nabla}=\mathbf{Z}^{i} \nabla_{i}$. Recalling the relation

$$
\beta_{i}^{j}=\partial_{i} Y^{j}+\Gamma_{i k}^{j} Y^{k}
$$

The first commutation can be reduced to the following form:

$$
\left[\dot{\tilde{\nabla}}^{(\vec{Y})}, \nabla_{\vec{C}}\right]=\left(\dot{\tilde{\nabla}}^{(\vec{Y})} C^{i}\right) \partial_{i} \phi
$$

The Trivial Solution can be obtained by stating that the two constant vectors are identical $\vec{T}=\vec{U} \rightarrow \vec{C}=\mathbf{0}$, however, any two vectors which satisfy $\nabla_{j} T^{i}=\nabla_{j} U^{i}=0$ and also satisfy:

$$
\dot{\tilde{\nabla}}^{(\vec{Y})} C^{i}=\dot{\tilde{\nabla}}^{(\vec{Y})}\left(U^{i}-T^{i}\right)=0
$$

Will also produce two Spatiotemporal Field Derivatives which commute. For simplicity, the trivial solution will be assumed; thus, the Spatiotemporal Field Derivative does indeed satisfy the commutation so long as two different directing vectors are not chosen, as per the following identity:

$$
\left[\ddot{\nabla}_{\sigma}^{\{\vec{T}\}}, \ddot{\nabla}_{\omega}^{\{\vec{T}\}}\right] \phi=0
$$

Obtaining the Spatiotemporal Field Derivative, and recalling the 3D analogue in Eq.(4), the basis vectors of the space can be obtained as:

$$
\boldsymbol{\zeta}_{\sigma}^{\{\vec{T}\}}=\ddot{\nabla}_{\sigma}^{\{\vec{T}\}} \mathbf{R}
$$

For brevity (and because it is implied by the $4 \mathrm{D}$ convention) the basis vectors may simply be referred to as $\boldsymbol{\zeta}_{\sigma}^{\{\vec{T}\}}$. This is explicitly stated as the following:

$$
\boldsymbol{\zeta}_{\sigma}^{\{\vec{T}\}}=\left(\begin{array}{ll}
\vec{T} & \mathbf{Z}_{i}
\end{array}\right) \quad, \quad \nabla_{j} T^{i}=0
$$

Any vector using these bases can be abbreviated into a new vector:

$$
\phi^{\sigma} \boldsymbol{\zeta}_{\sigma}^{\{\vec{T}\}}=\left(\begin{array}{ll}
\vec{T} & \mathbf{Z}_{i}
\end{array}\right)\left(\begin{array}{c}
\phi^{0} \\
\phi^{i}
\end{array}\right)=\phi^{0} \vec{T}+\phi^{i} \mathbf{Z}_{i}, \quad \nabla_{j} T^{i}=0
$$


All these terms can be projected onto a 3 vector by the following:

$$
\phi^{\sigma} \boldsymbol{\zeta}_{\sigma}^{\{\vec{T}\}}=\left(\phi^{0} T^{i}+\phi^{i}\right) \mathbf{Z}_{i}, \quad \nabla_{j} T^{i}=0
$$

Thus since vectors are normally $\vec{\phi}=\phi^{i} \mathbf{Z}_{i}$, this approach to our 4 space indicates that the definition of a vector in the $4 \mathrm{D}$-space is a similar vector in $3 \mathrm{D}$ off-set by a vector which only has as its requirement that $\nabla_{i} T^{j}=0$. If we consider time to be a fourth dimension, this parametrization outlines a $4 \mathrm{D}$ Vector Field projected into a 3D space.

\section{Higher Order Continuum Temporal Differ- ential Calculus}

The Temporal Field Derivative obtained in Eq.(81) has much utility:

$$
\dot{\tilde{\nabla}}_{[\vec{T}]}^{(\vec{Y})} \phi=\partial_{t} \phi+\left(T^{j}-Y^{j}\right) \nabla_{j} \phi, \quad \nabla_{j} T^{i}=0
$$

The Temporal Field Derivative can have higher-order analogues developed which will find application to practical situations.

\subsection{Higher Order Temporal Derivatives}

Due to the underlying symmetry in the Temporal Field Derivative, iterating the operator twice implies that a new Tensorially invariant operator is created:

$$
\left(\dot{\tilde{\nabla}}^{2}\right)_{\left[\vec{T}_{1}, \vec{T}_{2}\right]}^{(\vec{Y})}=\dot{\bar{\nabla}}_{\left[\overrightarrow{T_{2}}\right]}^{(\vec{Y})} \dot{\bar{\nabla}}_{\left[\vec{T}_{1}\right]}^{(\vec{Y})}
$$

Once again, in order to preserve full generality, two different tensors will be chosen, $\vec{T}$ and $\vec{U}$. In some cases, both tensors need to be identical which be denoted as follows:

$$
\left(\dot{\nabla}^{2}\right)_{\left[\vec{T}_{1}\right]}^{(\vec{Y})}=\left(\dot{\tilde{\nabla}}^{2}\right)_{\left[\vec{T}_{1}, \vec{T}_{1}\right]}^{(\vec{Y})}=\dot{\nabla}_{\left[\vec{T}_{1}\right]}^{(\vec{Y})} \dot{\nabla}_{\left[\vec{T}_{1}\right]}^{(\vec{Y})}
$$

It is worthy to note that since each Temporal Field Tensor is tensorial with respect to a tensor of rank $(\mathrm{k}, \mathrm{m})$, the Second-Order iteration is also tensorial with respect to the Spatiotemporal Field being operated on. 


\subsection{Truly Invariant D'Alembertian}

The D'Alembertian can be formulated to have an analogue under a dynamic variation of the coordinate system. Historically, the D'Alembertian is given by:

$$
\square=-\frac{1}{c^{2}} \partial_{t}^{2}+\nabla_{i} \nabla^{i}
$$

This operator can be found formed within the framework of Special Relativity as a Tensorial operator prescribed with the Minkowski Metric in 4D Spacetime [7]. Though this is an apt abbreviation, once again, issues arise when considering the invariance of the operator.

A generalization within the framework proposed by this paper is attempted to be obtained for an analogue of the D'Alembertian that remains invariant under a temporal distortion of the Coordinate Frame. Utilizing the Dual-Iteration of the Temporal Field Derivative from Eq.(96), the D'Alembertian can be reformulated as the following:

$$
\dot{\tilde{\square}}_{\left[\vec{T}_{1}, \vec{T}_{2}\right]}=-\frac{1}{c^{2}}\left(\dot{\tilde{\nabla}}^{2}\right)_{\left[\vec{T}_{1}, \vec{T}_{2}\right]}^{(\vec{Y})}+\nabla_{i} \nabla^{i}
$$

This definition can be simplified by recalling the definition of the Spatiotemporal Field Derivative obtained in the previous section from Eq.(84) and modifying it by a constant to re-use it as:

$$
\ddot{\nabla}_{\sigma}^{\{\vec{T}\}}=\left(\begin{array}{c}
\frac{1}{c} \dot{\tilde{\nabla}}_{[\vec{Y})}^{(\vec{T}]} \\
\nabla_{i}
\end{array}\right), \text { where } \nabla_{i} T^{j}=0
$$

The Minkowski Metric will also be utilized as defined by the following:

$$
\eta^{\mu \nu}=\left(\begin{array}{cccc}
-1 & 0 & 0 & 0 \\
0 & 1 & 0 & 0 \\
0 & 0 & 1 & 0 \\
0 & 0 & 0 & 1
\end{array}\right)
$$

Using these two definitions, the D'Alembertian from earlier can be re-written in a Tensorial Form:

$$
\dot{\tilde{\square}}_{\left[\vec{T}_{1}, \vec{T}_{2}\right]}=\eta^{\mu \nu} \ddot{\nabla}_{\mu}^{\left\{\vec{T}_{2}\right\}} \ddot{\nabla}_{\nu}^{\left\{\vec{T}_{1}\right\}}
$$

This D'Alembertian is fuly tensorial can can be used for several operations re-writing existing laws to account for a dynamic variation in the coordinate frame. 


\section{Applications \& Extensions of the Temporal Field Derivative}

The proposed operators and relations in this paper are seen to have multiple applications to correcting many physical theories.

\subsection{Wave Dynamics}

In light of the definition of the D'Alembertian in the previous section from Eq.(102), there can be a definition made for the well known Wave Equation in an ambient space of dimension $N$. Using the above operators, for an wave moving in a coordinate system that is under dynamic peturbation; its amplitude, $\phi$ will obey the law under full generality:

$$
\dot{\tilde{\square}}_{\left[\vec{T}_{1}, \vec{T}_{2}\right]} \phi=0
$$

This automatically implies all types of symmetries in the Law, as the Operator will preserve the Tensorial Nature of the Field under the two tensors, $\vec{T}_{1}$ and $\vec{T}_{2}$, each with $N$ degrees of freedom. Thus the law contains $2 N$ degrees of freedom more as it would have otherwise by the standard definition found in literature:

$$
\dot{\tilde{\square}}_{[\overrightarrow{0}, \overrightarrow{0}]} \phi=0
$$

The general Wave Equation from Eq.(103), when expanded under no dynamic variation of the coordinate system yields the following:

$$
\dot{\tilde{\square}}_{[\overrightarrow{0}, \overrightarrow{0}]} \phi=-\frac{1}{c^{2}} \partial_{t}^{2} \phi+\nabla_{i} \nabla^{i} \phi=0
$$

Returning the standard wave equation. Thus the alteration to the law only occurs when there is a slight spatiotemporal distortion of the coordinate system.

\subsection{Continuum Mechanics}

In the earlier representation of the physical law seen in Eq.(1), the speed of a continuum is given by $v^{i}$. This speed is actually a time derivative of the position coordinate which does transform like a tensor:

$$
v^{i}=\partial_{t} x^{i}
$$

In order to fully create a tensorial theory, the above speed must be reformulated tensorially (ie. $v^{i}=\dot{\tilde{\nabla}}_{[\vec{T}]}^{(\vec{Y})} x^{i}$ ), then incorporated into the general 
equation. Thus, we can restate the Continuum Mechanics Law of Motion under a dynamic distortion of the coordinate system as:

$$
\rho\left(\dot{\tilde{\nabla}}^{2}\right)_{\left[\vec{T}_{1}, \vec{T}_{2}\right]}^{(\vec{Y})} x^{i}=\rho b^{i}+\nabla_{j} \sigma^{j i}
$$

Eq.(106) is identical to the original linear theory in Eq.(1) except for the change in the Acceleration Term $\left(\left(\right.\right.$ ie. $\left.\rho \partial_{t} v^{i} \rightarrow \rho\left(\dot{\tilde{\nabla}}^{2}\right)_{\left[\vec{T}_{1}, \vec{T}_{2}\right]}^{(\vec{Y})} x^{i}\right)$. Most importantly, this equation is now fully compatible tensorially with all conventions. In a coordinate change all terms from Eq.(106) will transform acordingly as:

$$
\rho J_{i^{\prime}}^{i}\left(\dot{\tilde{\nabla}}^{2}\right)_{\left[\vec{T}_{1}, \vec{T}_{2}\right]}^{(\vec{Y})} x^{i^{\prime}}=\rho J_{i^{\prime}}^{i} b^{i^{\prime}}+J_{i^{\prime}}^{i} \nabla_{j^{\prime}} \sigma^{j^{\prime} i^{\prime}}
$$

Once the Jacobians have cancelled out, all the terms are identical once more in a different dynamic coordinate frame:

$$
\rho\left(\dot{\tilde{\nabla}}^{2}\right)_{\left[\vec{T}_{1}, \vec{T}_{2}\right]}^{(\vec{Y})} x^{i^{\prime}}=\rho b^{i^{\prime}}+\nabla_{j^{\prime}} \sigma^{j^{\prime} i^{\prime}}
$$

This is the implication when using the above Temporal Field Derivative

\subsection{Quantum Mechanics}

In accordance with the above theory, we can use the D'Alembertian Operator from Eq.(102) to form the Quantum Mechanical Schrodinger Equation previously stated in Eq.(2) in fully tensorial terms. Assuming the Wavefunction $\Psi$ is a Scalar Field (a rank 0 tensor), we obtain a restatement of the Schrodinger Equation:

$$
i \hbar \dot{\tilde{\nabla}}_{[\vec{T}]}^{(\vec{Y})} \Psi(Z, t)=\left(-\frac{\hbar^{2}}{2 \mu} \nabla_{i} \nabla^{i}+V\right) \Psi(Z, t)
$$

This equation is in accordance with the dynamic motion of a coordinate system and is also tensorial due to the tensorial invariant nature of $\Psi$. It also contains 3 degrees of freedom in the vector $\vec{T}$ recalling that the only condition on the vector is that $\nabla_{i} T^{j}=0$. This variation will affect Eq.(109) and therefore the Wavefunction Solution [12]. However, if it is assumed that $\vec{T}=\mathbf{0}$, and that $\vec{Y} \approx 0$, the equation reduces back to the classical Schrodinger's Equation from Eq.(2) as will the wavefunction of a particle [12]. Thus, the equation reflects that depending on how extreme the distortion of the coordinate space will be, the wave function of a particle will be altered. This may be useful for studying Quantum Mechanics near areas in space of large deformations which vary in time. 


\subsubsection{The Klein Gordon Equation}

The Klein Gordon Equation is an equation with structural ties to the Dirac and Schrodinger Equation, it contains the following form [12]:

$$
\left(-\frac{1}{c^{2}} \partial_{t}^{2}+\nabla_{i} \nabla^{i}\right) \psi=\frac{m^{2} c^{2}}{h^{2}} \psi
$$

It too can be altered by dynamic effects in the Coordinate System and as such will be invalid tensorially. Eq.(110) noticibly contains a D'Alembertian within the parentheses so re-stating it using the D'Alembertian outlined in Eq.(102) is easy. Formulating it as such results it the equation being Tensorially Invariant:

$$
\left(\dot{\tilde{\square}}_{\left[\vec{T}_{1}, \vec{T}_{2}\right]}-\frac{m^{2} c^{2}}{\hbar^{2}}\right) \psi=0
$$

This equation suggests $2 N$ degrees of freedom with the precise stating of the components of $\vec{T}_{1}$ and $\vec{T}_{2}$ only bound by the fact that

$$
\nabla_{j}\left(\vec{T}_{1}\right)^{i}=\nabla_{j}\left(\vec{T}_{2}\right)^{i}=0
$$

This modelling may serve interesting insights into the mechanics behing waves in the Physical Domain and in the Quantum Domain. [5, 12]

\section{Conclusion}

Physics and all its subdisciplines contains powerful insights on the mechanics of Nature around us; it has proposed several models of use ranging from the Schrodinger Equation to General Relativity [7, 12] and all theorems in between. [6]

Every single year presents an opportunity for a discovery to re-shape the manner the world is understood through Physics. Some discoveries confirm and enhance theorems such as the significance of the Gravitational Wave discovery and how it confirmed several postulates put forward by General Relativity [8]; however some discoveries demand the way Physics is fundamentally understood to change.

As with all theories in Physics: theorems which attempt to understand the fabric of reality and utilize Tensor Calculus to do so, must be adaptable. These theorems must be capable of adapting to the concept that Tensor Calculus like any other foundation in Mathematics is ultimately based on assumptions made about the space these theorems preside over. In cases where 
there is a dynamic distortion of the coordinate system, the Tensor Calculus used must be adapted for such a disturbance.

This paper has put forward a introductory foundation to Tensor Calculus utliized in a coordinate system which is under a dynamic distortion drawing inspiration from similar fields such as the Calculus of Moving Surfaces (CMS). It has established transformation laws for Tensors within these regions and has established Operators which enforce a Tensorial Transformation within a Dynamically Moving coordinate system.

This frame work can be utilized to observe how Physical theories change to accomodate for a distorting coordinate system such as with established theorems $[6,7,12]$ and can be utliized to develop new theoretical models which account for this temporal distortion to gain a better understanding of Physics as a whole.

This framework can also be utilized for coordinate systems which are in dynamic distortion constantly. Several examples in Biology have demonstrated such a variance $[10,11]$. Thus it is fathomable that this framework will find use for generating models of Biological Phenomena too.

\section{References}

[1] Grinfeld P. Introduction to Tensor Analysis and the Calculus of Moving Surfaces. New York: Springer, 2010. Print.

[2] Simmonds JG. A Brief on Tensor Analysis. New York: Springer-Verlag, 1994. Print.

[3] Thomas TY. Tensor Analysis and Differential Geometry. London: Academic Press Inc., 1961. Print.

[4] Willmore TJ. An Introduction to Differential Geometry. Oxford: Oxford University Press, 1998. Print.

[5] Liseikin VD. Grid Generation Methods. Dordrecht: Springer, 2010. Print.

[6] Gonzalez O, Stuart AM. A First Course in Continuum Mechanics. Cambridge: Cambridge University Press, 2008. Print.

[7] Misner CW, Thorne KS, Wheeler JA. Gravitation. San Francisco: W.H. Freeman and Company, 1973. Print. 
[8] Abbott et al. Observation of Gravitational Waves from a Binary Black Hole Merger. Physical Review Letters, 116, (2016): 061102

[9] Hurt R. "Gravitational Waves". LIGO Caltech, LIGO, July 10, 2015, ligo.caltech.edu

[10] Afas KC, Moschandreou TE. Analytic Multiplicative Separation and Existence Investigation of Non-Linear Oxygen Transport with Poiseuille Hemodynamic Flow in a Semi-Generalized Coordinate System. International Journal of Differential Equations and Applications, 14, (2015): No.4.

[11] Dembo M. Field Theories of the Cytoplasm. Comments on Theoretical Biology, 1, (1989): 159-177

[12] Gross F. Relativistic Quantum Mechanics and Field Theory. New Jersey: Wiley-VCH, 1993, Print. 